Artículo científico

Volumen 32(3):949-962 Septiembre-diciembre, 2021 e-ISSN 2215-3608, doi:10.15517/am.v32i3.42883

https://revistas.ucr.ac.cr/index.php/agromeso/index

\title{
Incorporación de proteína de suero dulce en un yogur batido bajo en grasa ${ }^{1}$
}

\section{Sweet whey protein addition to a low-fat yogurt}

\author{
Rebeca López-Calvo ${ }^{2 *}$,Diana Víquez-Barrantes ${ }^{2}$,Tatiana Araya-Arce ${ }^{3}$
}

1 Recepción: 11 de agosto, 2020. Aceptación: 25 de enero, 2021. Este trabajo forma parte de la Tesis de Licenciatura en Tecnología de Alimentos del tercer autor, como parte del proyecto 734-A6-057 inscrito en la Vicerrectoría de Investigación, Universidad de Costa Rica. San José, Costa Rica.

2 Universidad de Costa Rica, Centro Nacional de Ciencia y Tecnología de Alimentos. San José, Costa Rica. Apartado 11501-2060. rebeca. lopez@ucr.ac.cr (https://orcid.org/0000-0002-0482-2912), diana.viquezbarrantes@ucr.ac.cr (autora para correspondencia, https://www.orcid. org/0000-0002-3788-3076).

3 Universidad de Costa Rica, Escuela de Tecnología de Alimentos. San José, Costa Rica. tatiaraya87@ gmail.com (https://orcid.org/0000-00030782-2683).

\section{Resumen}

Introducción. El lacto suero de leche bovina es fuente de nutrientes, por lo que su aprovechamiento beneficiaría al consumidor y ayudaría a disminuir las consecuencias ambientales de su descarte. En Costa Rica, la mayor parte de este se utiliza como alimento para animales o se desecha en ríos y suelos. Es necesario desarrollar alternativas para su aprovechamiento. Objetivo. Evaluar el efecto de la incorporación de proteína sérica sobre las características físicas, contenido de aminoácidos y comportamiento en el almacenamiento del yogur con proteína de suero. Materiales y métodos. El estudio se desarrolló en las instalaciones de la Universidad de Costa Rica durante 2011-2015. El suero lácteo se acidificó hasta un $\mathrm{pH}$ de 4,8 , se calentó a $90{ }^{\circ} \mathrm{C}$ y se mantuvo en reposo por 90 min para lograr la precipitación de la proteína. Se desarrollaron dos muestras de yogur batido bajo en grasa, una control y una con proteína extraída del suero dulce. Se determinó la viscosidad, sinéresis, pH, macronutrientes, aminoácidos totales y libres, para ambos yogures. Se evalúo el comportamiento del yogur con proteína añadida y el agrado general durante veinticuatro días de almacenamiento, mediante análisis sensorial. Resultados. Se obtuvo un yogur con proteína añadida en el cual no se encontraron diferencias significativas, con respecto al yogur control, para pH, sinéresis, macronutrientes, aminoácidos libres, aminoácidos totales (a excepción de la treonina), interacción sinéresis-tiempo de almacenamiento y agrado general durante el almacenamiento. Conclusión. La calidad nutricional y la funcionalidad del yogur con proteína añadida se mantuvo con respecto al control, lo que permitiría incorporar proteína de suero con una metodología factible a escala artesanal.

Palabras clave: productos lácteos, aminoácidos, subproductos de la leche.

\begin{abstract}
Introduction. Bovine milk whey is a source of nutrients, so its use would benefit the consumer and help reduce the environmental impact of discarding it. In Costa Rica, most of it is destined as animal feed or disposed in rivers and soils. It is necessary to develop sustainable alternatives for its use. Objective. To evaluate the effect of serum


protein incorporation on the physical characteristics, amino acid content, and storage behavior of yogurt with whey protein. Materials and methods. The study was developed at the facilities of the Universidad de Costa Rica, during 2011-2015. The whey was acidified to a $\mathrm{pH}$ of 4.8 , heated to $90{ }^{\circ} \mathrm{C}$ and kept at rest for 90 min to achieve protein precipitation. Two samples of low-fat whipped yogurt were developed, one control and one with protein extracted from sweet whey. Viscosity, syneresis, $\mathrm{pH}$, macronutrient, total and free amino acids were determined for both yogurts. The behavior of the yogurt with added protein and the overall palatability were evaluated during twentyfour days of storage were evaluated by sensory analysis. Results. A yogurt with added protein showed no significant differences, in comparison with control yogurt, for $\mathrm{pH}$, syneresis, macronutrients, free amino acids, total amino acids (with exception for threonine), syneresis - storage time interaction and general pleasantness during storage. Conclusion. The nutritional quality and functionality of yogurt with added protein was maintained with respect to the control, which would allow the incorporation of whey protein with a feasible methodology on an artisanal scale.

Keywords: dairy products, amino acids, milk byproducts.

\section{Introducción}

En el 2016, la producción de leche en Costa Rica fue 1135 millones de litros, de los cuales 646 millones se utilizaron para ser procesados a nivel industrial y, de esta cantidad, un $18 \%$ se utilizó para producir queso (Madriz, 2017).

El queso es un alimento que está incluido en uno de los principales subgrupos de gasto a nivel nacional, que, junto con el pan, los cereales, la carne, la leche, los huevos, las legumbres y las hortalizas, representan el $65 \%$ del total del gasto per cápita en alimentos y bebidas no alcohólicas (Instituto Nacional de Estadística y Censos [INEC], 2014). En Costa Rica, se produce más el queso fresco, ya que, de acuerdo con el Nielsen Retail Index CAM (Amador et al., 2016), este representa un 54,2\% de preferencia por parte del consumidor.

Un 80-90 \% de la leche utilizada como materia prima en la producción de queso fresco se transforma en suero, el cual es desechado en su gran mayoría, lo que provoca un serio problema ambiental, debido a su alta capacidad contaminante, al tener una demanda bioquímica de oxígeno (DBO) de 30000 a $50000 \mathrm{mg}$ de oxígeno por litro, ácido láctico, minerales, sólidos suspendidos totales y un pH de 3,3 a 9,0 (Prazeres et al., 2012; Tetra Pak, 2015). En este contexto, se planteó evaluar el efecto de incorporar proteína extraída de suero dulce sobre las características físicas, químicas y sensoriales de un yogur bajo en grasa, con un método accesible para pequeños productores. Al ser un subproducto de la elaboración de queso, su composición puede variar según proceso específico del que se deriva. Una comparación efectuada entre la composición del suero dulce y el suero ácido obtenido de leche bovina, mostró que la principal diferencia, razón de su clasificación, es su pH, el cual es mayor a 5,6 para el primero y menor a 5,1 para el segundo (Smithers, 2015).

Otro factor negativo de la práctica de desechar el suero, es que no se aprovecha su alto valor nutritivo, ya que, por ejemplo, contiene compuestos bioactivos que tienen un efecto positivo en la salud de las personas. Estudios recientes han demostrado que algunos de estos compuestos protegen contra la hipertensión, debido a que tienen un efecto inhibidor sobre la actividad de la enzima transformadora de la angiotensina (ACE, por sus siglas en inglés) y esto ayuda a controlar la presión alta en la sangre, debido a que dilata los vasos sanguíneos y permite un mejor flujo de esta (Michelke et al., 2017). Se destaca que, de manera general, el suero contiene un $50 \%$ del total de los sólidos de la leche, e incluye la lactosa, seroproteínas, minerales (potasio, calcio, fósforo, sodio y magnesio) y un porcentaje de grasa (Arce-Méndez et al., 2016; Parra, 2009). 
Las proteínas del suero lácteo tienen un elevado valor biológico y un rico perfil de aminoácidos; son únicas, debido a que poseen propiedades moduladoras del sistema inmunológico (Mathai et al., 2017). Además, tienen un efecto positivo sobre el metabolismo de la glucosa, lo que se atribuye a la sensación de saciedad que generan. Se ha observado también una reducción de problemas inflamatorios, estrés oxidativo e hipertensión, gracias a su consumo (Sousa et al., 2012).

Para el aprovechamiento del suero lácteo existen diferentes tecnologías, la utilización de filtración por membrana ha tomado gran importancia, ya que tiene una serie de ventajas, entre ellas, la recuperación de fracciones de interés que pueden ser utilizadas en la industria alimentaria, con el fin de aprovechar su valor y obtener productos de buenas características (Prazeres et al., 2012), sin embargo, requieren una alta inversión inicial, espacio y representan dificultades en su limpieza.

En Costa Rica, se calcula que un $40 \%$ de la producción total de leche se transforma en el circuito artesanal, de este porcentaje, un $18 \%$ es convertido en queso (González, 2013), por lo que opciones tecnológicas para el aprovechamiento de suero lácteo en este tipo de producción se hacen necesarias.

Los quesos y el yogur fueron los lácteos de mayor crecimiento en ventas a nivel centroamericano en el período comprendido entre el 2015 y 2018 (Céspedes, 2018). Costa Rica es el país de Centroamérica donde un mayor porcentaje de tiendas ofrecen yogur $(82 \%)$, con un $31 \%$ del crecimiento de este producto en la región, un crecimiento en ventas de 10,6\% para los tipos no dietéticos y un 6,8\% para los tipos dietéticos, entre los años 2015 y 2016. Entre los años 2014 y 2016, el \% peso productos nuevos a total yogur se ha incrementado de 3,2 a 10,8 (Amador et al., 2016).

Formular un yogur con proteína extraída del suero dulce constituiría una alternativa para aprovechar el suero lácteo y mejorar el valor nutricional de un producto que, por sí solo, se considera como saludable y de alto consumo.

El objetivo de este trabajo fue evaluar el efecto de la incorporación de proteína sérica sobre las características físicas, contenido de aminoácidos y comportamiento en el almacenamiento del yogur con proteína de suero.

\section{Materiales y métodos}

\section{Período experimental}

El período experimental comprendió los años 2011 y 2013.

\section{Localización}

El desarrollo del yogur con proteína de suero, los análisis químicos proximales y el estudio con consumidores, se desarrolló en el Centro Nacional de Ciencia y Tecnología de Alimentos (CITA) y la determinación de aminoácidos se llevó a cabo en el Centro de Investigación en Nutrición Animal (CINA), ambos de la Universidad de Costa Rica, San José, Costa Rica.

\section{Materiales}

Para el desarrollo de los análisis se elaboraron dos muestras de yogur batido bajo en grasa, una muestra control y una con proteína extraída del suero dulce incorporada. Para ambas muestras se partió de leche entera de vaca, la cual se descremó como se indica en la Figura 1.

Los ingredientes utilizados fueron: leche descremada en polvo, estabilizante para yogur, azúcar, cultivo para yogur, saborizante de fresa. 


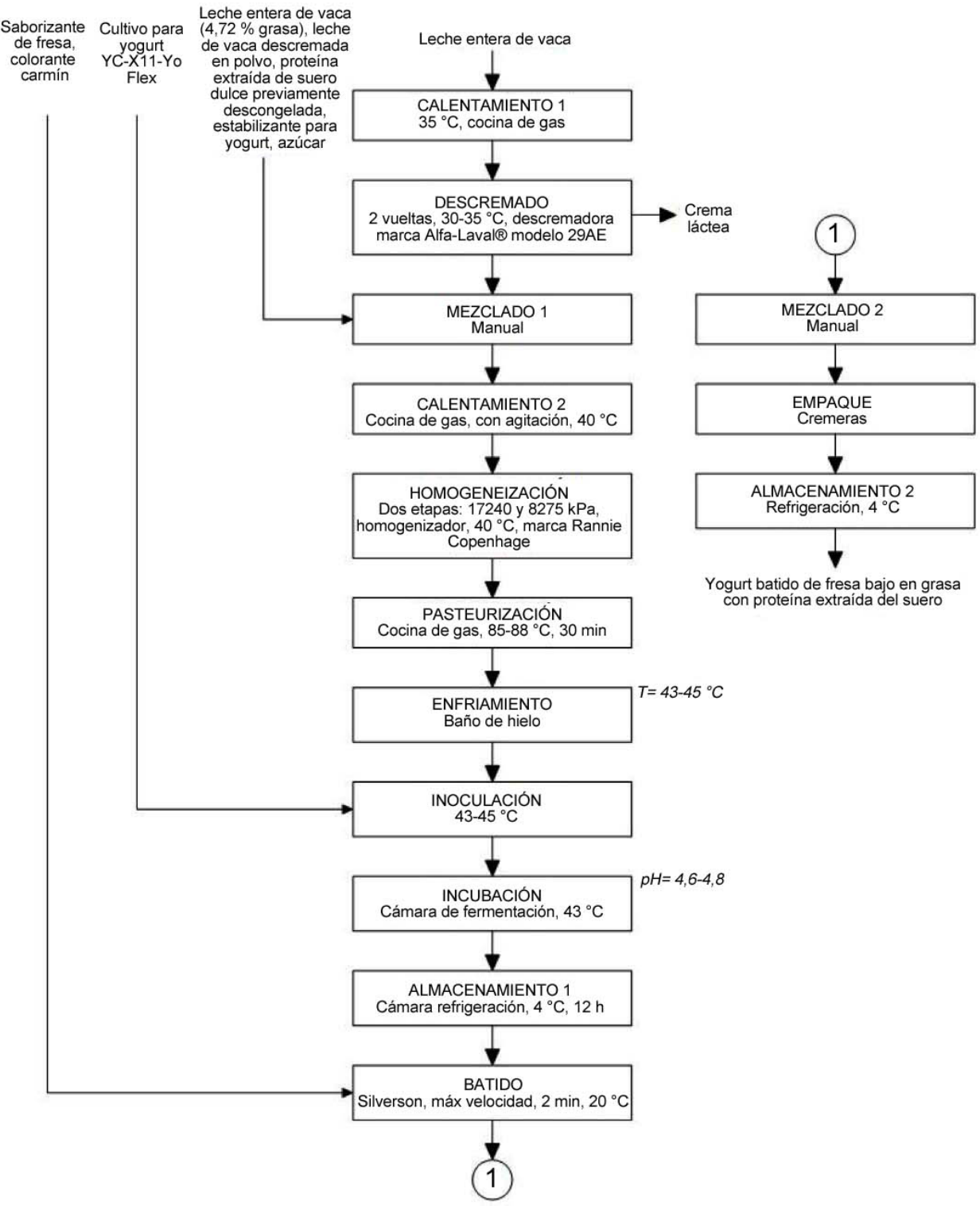

Figura 1. Flujo de proceso para la elaboración de un yogur con proteína extraída del suero dulce. Centro Nacional de Ciencia y Tecnología de Alimentos (CITA), Universidad de Costa Rica, San José, Costa Rica. 2013.

Figure 1. Process flow for the elaboration of a yogurt with protein extracted from sweet whey. Centro Nacional de Ciencia y Tecnologia de Alimentos (CITA), Universidad de Costa Rica, San Jose, Costa Rica. 2013.

A la muestra con proteína añadida se le incorporó el precipitado de proteína extraída del suero dulce (Figura 2). Cada yogur se formuló para obtener un contenido similar de proteína $(3,7 \%)$; se tomó como base la indicación del Reglamento Técnico RTCR 414:2008 Yogurt para consumo directo, en el que se establece que el contenido mínimo de proteína debe ser de $2,7 \mathrm{~g}$ de proteína por $100 \mathrm{~g}$ de producto. 


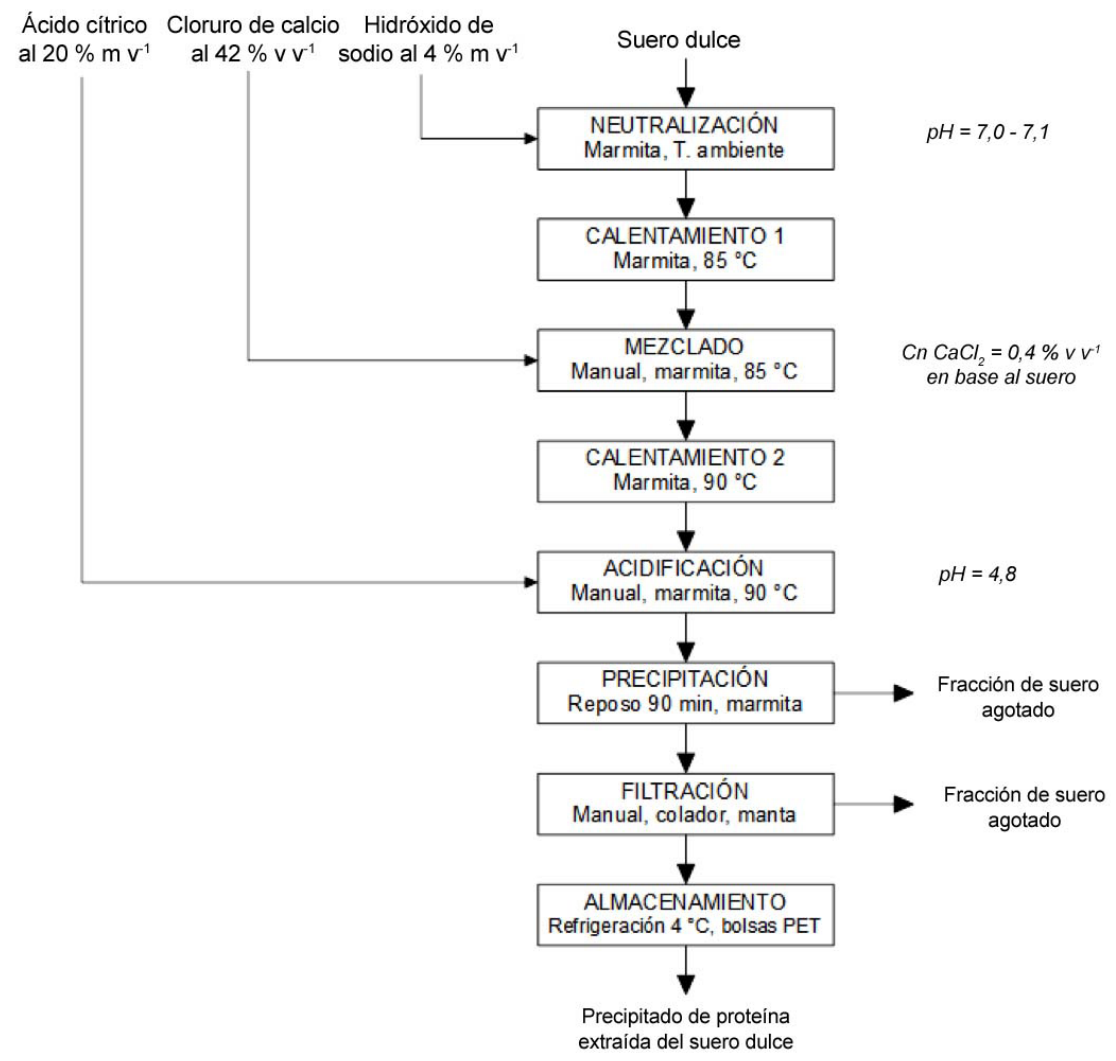

Figura 2. Proceso de obtención de proteína de suero lácteo. Centro Nacional de Ciencia y Tecnología de Alimentos (CITA), Universidad de Costa Rica, San José, Costa Rica. 2013.

Figure 2. Process followed to obtain whey protein. Centro Nacional de Ciencia y Tecnologia de Alimentos (CITA), Universidad de Costa Rica, San Jose, Costa Rica. 2013.

\section{Extracción de la proteína de suero}

Para la extracción de la proteína se realizó un proceso de acidificación y precipitación a partir del suero dulce que se describe en la Figura 2.

\section{Elaboración del yogur}

La formulación del yogur batido de fresa bajo en grasa, se definió mediante pruebas preliminares y se realizó con base en la composición de sólidos no grasos (SNG), sólidos grasos (SG) y sólidos totales (ST) de un yogur batido bajo en grasa reportado en la literatura. Asimismo, se tomó en cuenta las especificaciones para un yogur semidescremado establecidas en la norma nacional para el yogur (yogurt) y el yogur azucarado (Figura 1) (Ministerio de Economía, Industria y Comercio, 2008). 


\section{Análisis fisicoquímicos}

Se midieron las siguientes características físicas del yogur: sinéresis, $\mathrm{pH}$ y viscosidad aparente; además, se determinó el contenido de lactosa, grasa, humedad y proteína, tanto en el yogur con proteína extraída del suero dulce como en el yogur control, en el día uno de almacenamiento.

Para cada tipo de yogur se determinó el contenido de los aminoácidos totales y libres que se enlistan a continuación: ácido aspártico, ácido glutámico, serina, histidina, glicina, treonina, arginina, alanina, tirosina, cisteína, valina, metionina, isoleucina, leucina y lisina.

La determinación de sinéresis expresada como el volumen de suero desprendido durante el almacenamiento, se basó en el método descrito por Rojas-Castro et al. (2007). Se pesaron muestras de $50 \mathrm{~g}$ de yogur en una balanza granataria, luego se centrifugaron a $3000 \mathrm{rpm}$ durante $20 \mathrm{~min}$, a $10^{\circ} \mathrm{C}$ en una centrífuga refrigerada. El total de suero desprendido se trasvasó luego a una probeta y se midió el volumen.

El pH se determinó por medio de un pHmetro.

La viscosidad aparente se midió con un viscosímetro de Brookfield y con el husillo R5, a $5 \mathrm{rpm}$ y a $6-7^{\circ} \mathrm{C}$, temperatura de almacenamiento del yogur (Rojas-Castro et al., 2007).

En el análisis de lactosa se siguió el método descrito por Sullivan \& Carpenter (1993).

Los métodos de humedad, grasa por Babcock y nitrógeno utilizados, fueron los indicados por la Sociedad Oficial de Química (AOAC por sus siglas en inglés) 990.19; 989.04 y 991.20, respectivamente (Association of Official Analytical Chemists, 2005).

Para la determinación de aminoácidos totales y libres, se utilizó cromatografía líquida (HPLC) con un detector de fluorescencia a $450 \mathrm{~nm}$ de emisión y $340 \mathrm{~nm}$ de excitación. Se realizó una derivatización pre-columna automatizada con o-ftalaldehído (OPA) para formar un compuesto fluorescente con cada aminoácido para que pudiera ser detectado. Se utilizó una columna de fase reversa Zorbax Eclipse-AAA de 4,6x150 m, 3,5 $\mu$ m código PN966400-902. El flujo de inyección fue de $2000 \mathrm{ml} \mathrm{min}{ }^{-1}$ y la temperatura de columna de $41{ }^{\circ} \mathrm{C}$.

\section{Estudio de almacenamiento}

Se realizó un estudio de almacenamiento del yogur desarrollado; el análisis se llevó a cabo de forma escalonada, para esto se almacenaron muestras en refrigeración a $4{ }^{\circ} \mathrm{C}$ durante $1,8,15,18$ y 22 días. Se evaluó la sinéresis, el $\mathrm{pH}$, la viscosidad aparente, el contenido de aminoácidos libres y el agrado general.

La evaluación del agrado del yogur se realizó por medio de una escala híbrida y un panel de cien jueces consumidores de yogur. Cada juez evaluó el nivel de agrado de las muestras en una escala de diez puntos que iba desde "me disgusta muchísimo" a "me gusta muchísimo".

\section{Análisis estadístico}

Se realizó un diseño de bloques con dos tratamientos y cinco repeticiones para comparar los promedios de los análisis físicos y químicos, con el fin de determinar si había o no diferencia significativa entre el yogur formulado y el control. Las repeticiones constituyeron los bloques del diseño y los tratamientos fueron: con proteína añadida y sin proteína añadida.

Se realizó un análisis de regresión lineal para determinar si existía algún tipo de relación entre cada una de las variables físicas medidas, así como el contenido de aminoácidos libres, con respecto al tiempo de almacenamiento. 
Los resultados de la evaluación sensorial se analizaron por medio de un ANOVA de un factor, con el objetivo de determinar si hubo o no diferencia significativa en el nivel de agrado entre las muestras. En caso de encontrar una diferencia significativa, se procedió a realizar una prueba de Tukey para determinar cuál o cuáles de las muestras tenían un nivel de agrado distinto.

Los análisis estadísticos correspondientes se llevaron a cabo con el programa estadístico JMP 4.0.4 con un nivel de significancia de $\alpha=0,05$.

\section{Resultados}

\section{Composición química del yogur con proteína añadida y del control}

El yogur con proteína añadida y el yogur control no presentaron diferencias significativas $(p<0,05)$ en cuanto a su composición química (Cuadro 1), lo que demuestra que el ajuste en la formulación del yogur con proteína añadida permitió mantener la concentración de este nutriente constante en ambas muestras.

Cuadro 1. Composición química del yogur con proteína añadida extraída de suero dulce y del yogur control. Centro Nacional de Ciencia y Tecnología de Alimentos (CITA), Universidad de Costa Rica, San José, Costa Rica. 2013.

Table 1. Chemical composition of yogurt with added protein extracted from sweet whey and yogurt control. Centro Nacional de Ciencia y Tecnologia de Alimentos (CITA), Universidad de Costa Rica, San Jose, Costa Rica. 2013.

\begin{tabular}{|c|c|c|c|c|}
\hline \multirow[t]{2}{*}{ Tratamiento } & Humedad & Proteína & Grasa & Lactosa \\
\hline & \multicolumn{4}{|c|}{$\left(\mathrm{g} 100 \mathrm{~g}^{-1}\right)$} \\
\hline Yogur con proteína añadida & $78,5 \pm 0,7^{\mathrm{a}}$ & $3,6 \pm 0,1^{\mathrm{a}}$ & $2,1 \pm 0,2^{\mathrm{a}}$ & $3,2 \pm 0,2^{\mathrm{a}}$ \\
\hline Yogur control & $77,4 \pm 3,2^{\mathrm{a}}$ & $3,8 \pm 0,7^{\mathrm{a}}$ & $2,2 \pm 0,3^{\mathrm{a}}$ & $3,3 \pm 0,5^{\mathrm{a}}$ \\
\hline
\end{tabular}

* Datos reportados como promedio \pm intervalo de confianza $(n=4)$, con un nivel de confianza del $95 \% / *$ Data reported as mean \pm confidence interval $(n=4)$, with $95 \%$ as a level of confidence.

(a) Valores entre columnas, seguidos por letras distintas, indican diferencia significativa entre los tratamientos (Tukey, $\mathrm{p}<0,05) \quad /(\mathrm{a}) \mathrm{Means}$ between in columns with different letters, are significantly different between treatments (Tukey, $\mathrm{p}<0,05)$.

Determinación del contenido de aminoácidos totales y libres en el yogur con proteína añadida y en el control

Los yogures preparados no presentaron diferencias significativas $(\mathrm{p}<0,05)$ en el perfil de aminoácidos libres (Cuadro 2), mientras que para el perfil de aminoácido totales el yogur control presentó un contenido de treonina significativamente mayor $(\mathrm{p}<0,05)$.

Propiedades físicas (viscosidad, sinéresis) del yogur con proteína añadida y del yogur control

El yogur con proteína añadida presentó una viscosidad significativamente mayor $(\mathrm{p}<0,05)$ a la del yogur control, pero no se encontraron diferencias significativas $(\mathrm{p}<0,05)$ en la sinéresis de los productos elaborados (Cuadro 3). 
Cuadro 2. Contenido de aminoácidos totales $\left(\mu \mathrm{mol} \mathrm{AA} \mathrm{mg}{ }^{-1}\right.$ de proteína) y aminoácidos libres $\left[\left(\mu \mathrm{mol} \mathrm{AA} \mathrm{mg}{ }^{-1}\right.\right.$ de proteína $\left.\left(x 10^{1}\right)\right]$ en el yogur con proteína añadida y en el yogur control. Centro Nacional de Ciencia y Tecnología de Alimentos (CITA) y Centro de Investigación en Nutrición Animal (CINA), ambos pertenecen a la Universidad de Costa Rica, San José, Costa Rica. 2013.

Table 2. Total amino acid content ( $\mu \mathrm{mol} \mathrm{AA} \mathrm{mg}{ }^{-1}$ of protein) and free amino acid content $\left[\left(\mu \mathrm{mol} \mathrm{AA} \mathrm{mg}{ }^{-1}\right.\right.$ of protein $\left.\left(\mathrm{x} 10^{1}\right)\right]$ on yogurt with added protein and yogurt control. Centro Nacional de Ciencia y Tecnologia de Alimentos (CITA) y Centro de Investigacion en Nutricion Animal (CINA), de la Universidad de Costa Rica, San Jose, Costa Rica. 2013.

\begin{tabular}{|c|c|c|c|c|}
\hline \multirow[t]{2}{*}{ Aminoácido } & \multicolumn{2}{|c|}{$\begin{array}{l}\text { Contenido de aminoácidos totales } \\
\left(\mu \mathrm{mol} \mathrm{AA} \mathrm{mg}^{-1} \text { de proteína }\right)\end{array}$} & \multicolumn{2}{|c|}{$\begin{array}{l}\text { Contenido de aminoácidos libres } \\
\left(\mu \mathrm{mol} \text { AA } \text { mg }^{-1} \text { de proteína }\left(\mathbf{x 1 0}^{1}\right)\right)\end{array}$} \\
\hline & $\begin{array}{c}\text { Yogur con proteína } \\
\text { añadida } \\
\end{array}$ & Yogur control & $\begin{array}{c}\text { Yogur con proteína } \\
\text { añadida } \\
\end{array}$ & Yogur control \\
\hline Ácido aspártico & $8,5 \pm 2,4^{\mathrm{a}}$ & $12,1 \pm 7,1^{\mathrm{a}}$ & N.D & N.D \\
\hline Ácido glutámico & $16,9 \pm 4,8^{\mathrm{a}}$ & $25,2 \pm 6,2^{\mathrm{a}}$ & $0,8 \pm 1,2^{\mathrm{a}}$ & $0,5 \pm 0,5^{\mathrm{a}}$ \\
\hline Serina & $5,7 \pm 2,3^{\mathrm{a}}$ & $8,0 \pm 0,4^{\mathrm{a}}$ & $0,6 \pm 1,0^{\mathrm{a}}$ & $0,5 \pm 0,8^{\mathrm{a}}$ \\
\hline Histidina & $0,3 \pm 0,1^{\mathrm{a}}$ & $0,3 \pm 0,1^{\mathrm{a}}$ & $0,2 \pm 0,2^{\mathrm{a}}$ & $0,3 \pm 0,4^{\mathrm{a}}$ \\
\hline Glicina & N.D & N.D & $1,4 \pm 1,5^{\mathrm{a}}$ & $2,4 \pm 2,4^{\mathrm{a}}$ \\
\hline Treonina & $0,8 \pm 0,4^{\mathrm{a}}$ & $1,1 \pm 0,1^{\mathrm{b}}$ & N.D & N.D \\
\hline Arginina & $3,7 \pm 1,6^{\mathrm{a}}$ & $5,1 \pm 0,1^{\mathrm{a}}$ & $1,3 \pm 1,1^{\mathrm{a}}$ & $1,5 \pm 1,2^{\mathrm{a}}$ \\
\hline Alanina & $0,3 \pm 0,1^{\mathrm{a}}$ & $0,3 \pm 0,2^{\mathrm{a}}$ & $2,4 \pm 1,1^{\mathrm{a}}$ & $2,2 \pm 0,7^{\mathrm{a}}$ \\
\hline Tirosina & $4,3 \pm 1,7^{\mathrm{a}}$ & $6,1 \pm 0,6^{\mathrm{a}}$ & N.D & N.D \\
\hline Cisteína & $1,8 \pm 0,9^{\mathrm{a}}$ & $1,8 \pm 0,8^{\mathrm{a}}$ & $4,6 \pm 2,3^{\mathrm{a}}$ & $3,8 \pm 3,8^{\mathrm{a}}$ \\
\hline Valina & $0,4 \pm 0,3^{\mathrm{a}}$ & $0,4 \pm 0,2^{\mathrm{a}}$ & $1,2 \pm 0,9^{\mathrm{a}}$ & $1,0 \pm 0,7^{\mathrm{a}}$ \\
\hline Metionina & $2,3 \pm 1,3^{\mathrm{a}}$ & $3,2 \pm 0,7^{\mathrm{a}}$ & $2,5 \pm 2,5^{\mathrm{a}}$ & $2,4 \pm 1,9^{\mathrm{a}}$ \\
\hline Isoleucina & $3,8 \pm 2,2^{\mathrm{a}}$ & $5,3 \pm 1,6^{\mathrm{a}}$ & $0,9 \pm 0,7^{\mathrm{a}}$ & $1,0 \pm 0,8^{\mathrm{a}}$ \\
\hline Leucina & $6,2 \pm 3,5^{\mathrm{a}}$ & $7,9 \pm 1,3^{\mathrm{a}}$ & N.D & N.D \\
\hline Lisina & $4,5 \pm 1,8^{\mathrm{a}}$ & $6,2 \pm 1,7^{\mathrm{a}}$ & $15,1 \pm 10,4^{\mathrm{a}}$ & $15,8 \pm 23,4^{\mathrm{a}}$ \\
\hline
\end{tabular}

* Datos reportados como promedio \pm intervalo de confianza $(n=4)$, con un nivel de confianza del $95 \%$. Nombres en cursiva corresponden a aminoácidos esenciales / $*$ Data reported as mean \pm confidence interval $(\mathrm{n}=4)$, with $95 \%$ as a level of confidence. Names in italic font indicates essential amino acids.

(a) Valores de los promedios entre columnas con letras distintas, indican diferencia significativa entre los tratamientos (Tukey, $\mathrm{p}<0,05)$. N.D: No determinado / (a) Values of the averages between columns with different letters, are significantly different between treatments (Tukey, $\mathrm{p}<0,05)$. N.D: not determined.

Cuadro 3. Propiedades físicas del yogur con proteína añadida y del yogur control. Centro Nacional de Ciencia y Tecnología de Alimentos (CITA), Universidad de Costa Rica. San José, Costa Rica. 2013.

Table 3. Physical properties of yogurt with added protein and yogurt control. Centro Nacional de Ciencia y Tecnologia de Alimentos (CITA), Universidad de Costa Rica. San Jose, Costa Rica. 2013.

\begin{tabular}{lcc}
\hline Tratamiento & Viscosidad $($ Pa・s) & Sinéresis $\left(\mathbf{g} \mathbf{1 0 0} \mathbf{g}^{-1}\right)$ \\
\hline Yogur con proteína añadida & $38,9 \pm 11,5^{\mathrm{a}}$ & $17,6 \pm 4,2^{\mathrm{a}}$ \\
Yogur control & $26,9 \pm 11,8^{\mathrm{b}}$ & $19,3 \pm 5,0^{\mathrm{a}}$ \\
\hline
\end{tabular}

*Datos son reportados como promedio \pm intervalo de confianza $(n=4)$, con un nivel de confianza del $95 \% / *$ Data reported as mean \pm confidence interval $(\mathrm{n}=4)$, with $95 \%$ as a level of confidence.

${ }^{(\mathrm{a}, \mathrm{b})}$ Valores de los promedios entre filas con letras distintas, indican diferencia significativa entre los tratamientos $($ Tukey, $\mathrm{p}<0,05) /(\mathrm{a}, \mathrm{b})$ Values of the averages between rows with different letters, are significantly different between treatments (Tukey, $\mathrm{p}<0,05)$. 


\section{Propiedades físicas, contenido de aminoácidos libres y agrado general del yogur con proteína añadida durante el almacenamiento $(\mathrm{t}=\mathbf{2 4}$ días)}

Se observó que la relación entre las variables tiempo de almacenamiento-viscosidad (Figura 3) y tiempo de almacenamiento-pH fue significativa $(\mathrm{p}<0,05)$, con una tendencia a aumentar para el primer caso y una tendencia a disminuir para el segundo. Mientras que la relación entre la sinéresis y el tiempo de almacenamiento (Figura 4) no fue significativa $(\mathrm{p}>0,05)$. La correlación entre el contenido de aminoácidos libres y el tiempo de almacenamiento solo fue significativa para la arginina, cisteína, valina, isoleucina y lisina $(\mathrm{p}<0,05)$. Los resultados demostraron que el agrado (Cuadro 4) de los panelistas no se vio afectado por el tiempo de almacenamiento ( $\mathrm{p}>0,05)$.

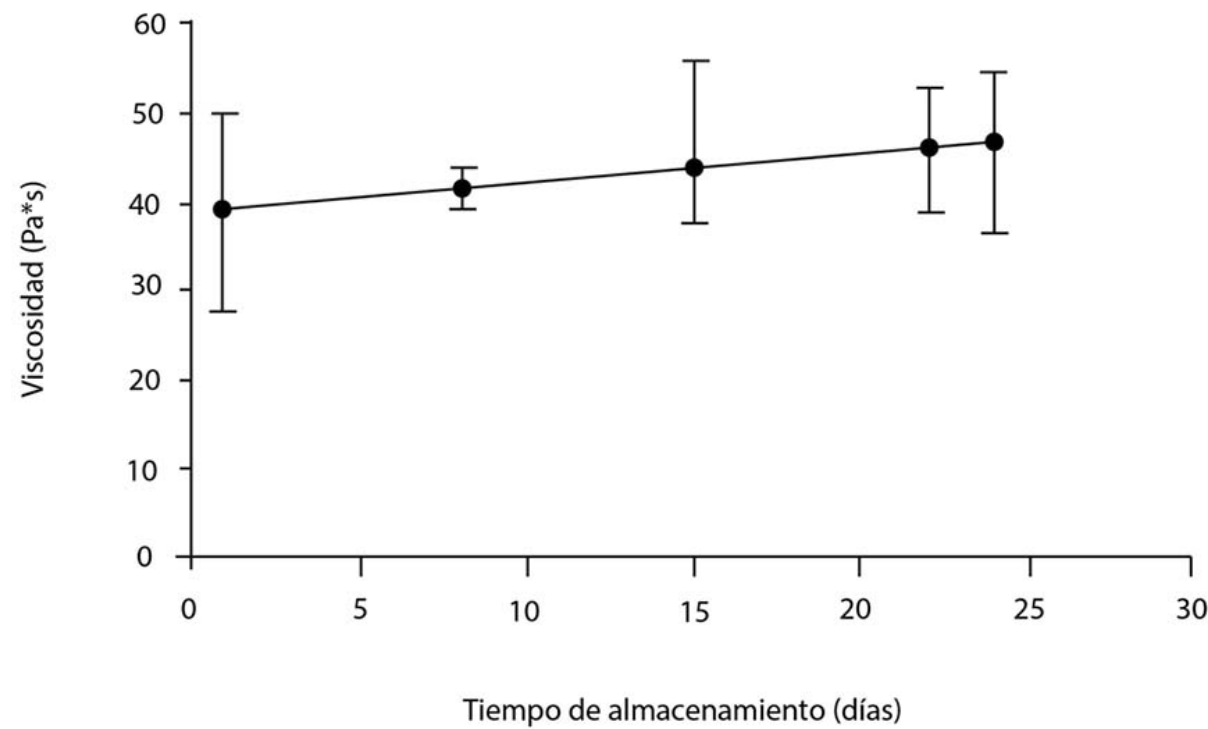

Figura 3. Comportamiento de la viscosidad aparente del yogur con proteína añadida, durante el tiempo de almacenamiento. Datos son reportados como promedios \pm el intervalo de confianza $(n=4)$, con un nivel de confianza del $95 \%$. Centro Nacional de Ciencia y Tecnología de Alimentos (CITA), Universidad de Costa Rica, San José, Costa Rica. 2013.

Figure 3. Behavior of the apparent viscosity of yogurt with added protein, during storage time. Data reported as means \pm the confidence interval ( $\mathrm{n}=4)$, with $95 \%$ as a level of confidence. Centro Nacional de Ciencia y Tecnologia de Alimentos (CITA), Universidad de Costa Rica, San Jose, Costa Rica. 2013.

\section{Discusión}

La calidad nutricional del yogur con proteína añadida se mantuvo al realizar una sustitución del $3 \%$ de leche por proteína de suero lácteo obtenida mediante calentamiento y acidificación, se aprovechó así un subproducto de la industria quesera. Una ventaja de este método de obtención de la proteína es la fácil implementación en producción artesanal.

El yogur con proteína añadida no presentó una mejora en el perfil de aminoácidos libres, si bien este resultado no era esperado (U.S. Dairy Export Council, s.f.; Walzem et al., 2002), se atribuyó a dos factores, primero la variabilidad del método, el error asociado a la magnitud reportada como intervalos de confianza fue alto, lo que 


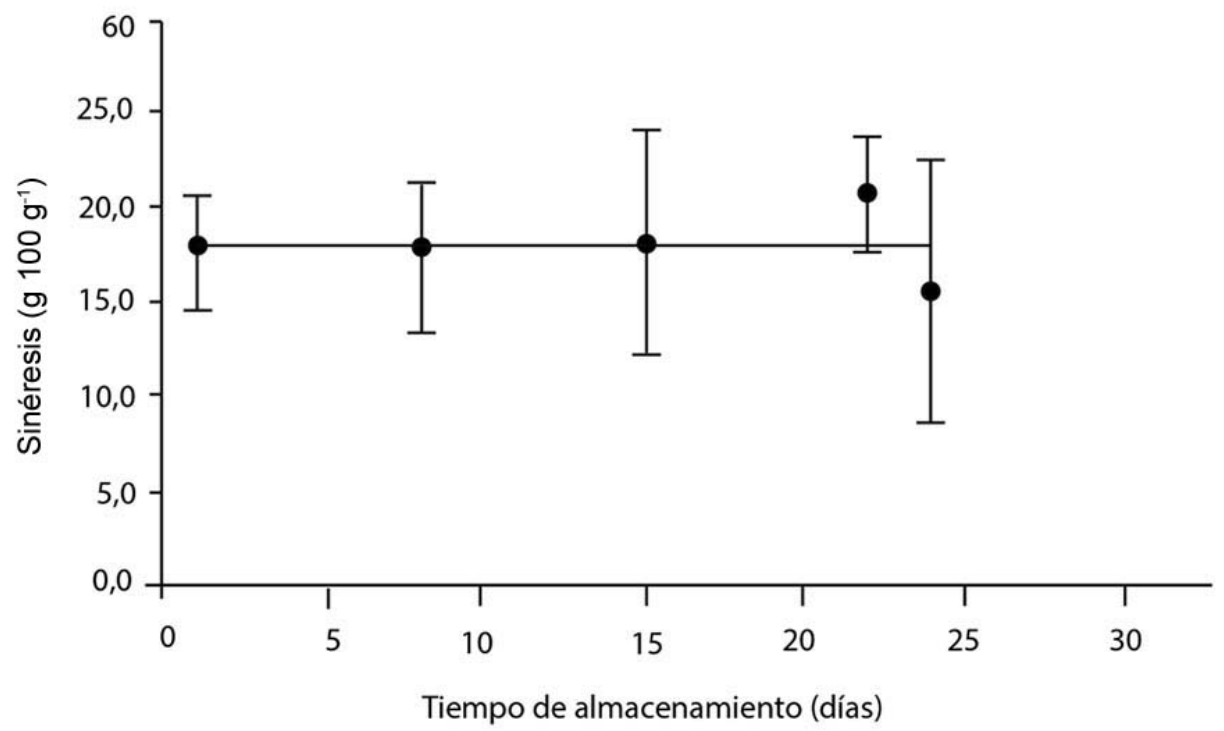

Figura 4. Comportamiento de la sinéresis del yogur con proteína añadida, durante el tiempo de almacenamiento. Datos son reportados como promedios \pm el intervalo de confianza $(n=4)$, con un nivel de confianza del $95 \%$. Centro Nacional de Ciencia y Tecnología de Alimentos (CITA), Universidad de Costa Rica, San José, Costa Rica. 2013.

Figure 4. Behavior of the syneresis of yogurt with added protein, during storage time. Data reported as means \pm the confidence interval $(\mathrm{n}=4)$, with $95 \%$ as a level of confidence. Centro Nacional de Ciencia y Tecnologia de Alimentos (CITA), Universidad de Costa Rica, San Jose, Costa Rica. 2013.

Cuadro 4. Agrado del yogur con proteína añadida, en distintos días de almacenamiento. Centro Nacional de Ciencia y Tecnología de Alimentos (CITA). Universidad de Costa Rica, San José, Costa Rica. 2013.

Table 4. Liking of yogurt with added protein, in different days of storage. Centro Nacional de Ciencia y Tecnologia de Alimentos (CITA). Universidad de Costa Rica, San Jose, Costa Rica. 2013.

\begin{tabular}{cc}
\hline Almacenamiento (días) & Agrado* $^{*}$ \\
\hline 1 & $7,2 \pm 0,4$ \\
15 & $7,2 \pm 0,4$ \\
18 & $7,0 \pm 0,4$ \\
22 & $7,1 \pm 0,4$ \\
\hline
\end{tabular}

*Datos reportados como promedios \pm intervalo de confianza $(n=4)$, con un nivel de confianza del $95 \% / *$ Data reported as means \pm confidence interval $(n=4)$, with $95 \%$ as a level of confidence.

hace posible que el error estándar impida observar una diferencia significativa y, segundo, el aporte total de proteína sérica añadido es del $0,2 \%$, por lo que se mantiene la caseína como la proteína predominante.

$\mathrm{Al}$ añadir proteína sérica obtenida por un método de fácil aplicación en producción artesanal a un producto de alto consumo como lo es el yogur, se planteó una buena opción para el aprovechamiento del suero lácteo y contribuir de esta manera a la disminución del impacto ambiental asociado a la producción de queso fresco, pero mantuvo la calidad nutricional del yogur que comúnmente se encuentra en el mercado.

Otros estudios realizados, en los cuales se adicionan concentrados de suero con un mayor contenido de proteína que el obtenido en el presente estudio, se logró una mejora de las características bioactivas del yogur, 
se mantuvieron las propiedades reológicas similares a las del yogur elaborado con ingredientes tradicionales (ElFattah et al., 2018; González-Martínez et al., 2002; Unal \& Akalın, 2012). Por lo que, para futuras investigaciones, se recomienda evaluar si dichos resultados se alcanzan al incorporar proteínas obtenidas por acidificación y precipitación, como se hizo en este estudio, sin etapas de concentración posterior.

El aumento en la viscosidad del yogur con proteína de suero coincidió con lo encontrado en otros estudios (Hugunin, 2009; Matumoto-Pintro et al., 2011; Sodini et al., 2005), sin embargo, es importante resaltar que, al utilizar proteína obtenida por acidificación y precipitación, el contenido de humedad presente en esta hizo necesario una mayor cantidad de estabilizante adicionada con respecto al yogur control, factor que también afecta la viscosidad del producto.

El estudio de almacenamiento durante 24 días, permitió observar un comportamiento esperado en la viscosidad y el $\mathrm{pH}$. La incorporación de la proteína sérica obtenida por acidificación y precipitación, mantuvo estas propiedades físicas sin interferir en las dinámicas de post-acidificación típicas de los productos fermentados similares a los yogures elaborados con ingredientes tradicionales.

Con respecto a la sinéresis, los resultados obtenidos fueron favorables para el desarrollo de un yogur comercializable, ya que mantuvo las características de calidad adecuadas por un período suficiente para su comercialización y consumo.

En el tiempo 0 del estudio de almacenamiento, se evidencia que la sinéresis no disminuyó en el yogur con proteína añadida, como lo reportaron otros autores (Bertrand et al., 2008; Hugunin, 2009), si bien los ingredientes del suero lácteo tienen un efecto de reducción de este tipo de defectos, la forma de obtención de esta influye en su funcionalidad. En el presente estudio se utilizó una temperatura de $90{ }^{\circ} \mathrm{C}$ para la recuperación de la proteína, Sodini et al. (2006) reportaron que, a temperaturas de obtención de $88^{\circ} \mathrm{C}$, la funcionalidad de la proteína sérica se vio afectada y disminuyó su capacidad de retención de agua. Mientras que en el estudio de almacenamiento se esperaba ver un aumento de la sinéresis en el tiempo (Zare et al., 2011), debido a la disminución de la capacidad de retención de agua de las proteínas, en el presente estudio se mantuvo constante durante los 24 días de almacenamiento.

Al extraer la proteína, con base en el proceso definido en el presente estudio, no hubo una variación en la sinéresis con respecto al yogur elaborado con ingredientes tradicionales, lo que permitió mantener las características constantes en el almacenamiento.

El yogur cuenta con bacterias lácticas, las cuales mantienen su actividad proteolítica durante el almacenamiento (González-Olivares et al., 2011), esto se pudo corroborar tanto con el comportamiento del pH en el tiempo, el cual tendió a disminuir, como con el aumento en la concentración de arginina, cisteína, valina, isoleucina y lisina. Es importante destacar que en un mayor tiempo de almacenamiento podrían observarse mayores concentraciones de aminoácidos libres.

Durante el tiempo de almacenamiento no se vio afectada la estabilidad ni la calidad del yogur con proteína añadida, lo que permitió establecer como parámetro al menos veintidós días de vida útil para este producto, pudiéndose extender si se adicionara un preservante a su formulación. Esto debido a que los panelistas percibieron los yogures de forma similar, sin importar el tiempo de almacenamiento (Cuadro 4).

\section{Conclusiones}

La calidad nutricional del yogur con proteína añadida se mantuvo con respecto al control.

La variabilidad encontrada en el método de análisis no permite concluir sobre el efecto de la incorporación de proteína en el perfil de aminoácidos.

Las características físicas del yogur formulado evaluadas durante el almacenamiento fueron convenientes para su comercialización, al mantener la viscosidad y pH esperados y mejorar el comportamiento en sinéresis. 
El producto formulado se adecuó a las últimas tendencias de consumo relacionadas con la salud, por lo que su inclusión en el mercado puede ser bien aceptada y su producción representa una opción de diversificación de productos para el sector artesanal que contribuye, además, a la reducción del impacto ambiental de la producción de queso.

\section{Referencias}

Amador, A., Marín, M., \& Pineda, P. (2016, Octubre 18-19). Decodificando el consumo de productos lácteos en la actualidad de Centroamérica, ¿Cómo ha evolucionado el gasto en los productos lácteos frente al crecimiento de la industria de consumo masivo? [Conferencia oral]. XXII Congreso Nacional Lechero, San José, Costa Rica. http://proleche.com/ recursos/documentos/congreso2016/Charla14.pdf

Arce-Méndez, J. R., Thompson- Vicente, E., \& Calderón-Villaplana, S. (2016). Incorporación de la proteína del suero lácteo en un queso fresco. Agronomía Mesoamericana, 27(1), 61-71. http://dx.doi.org/10.15517/am.v27i1.21878

Association of Official Analytical Chemists (2005). Official methods of analysis (18 ${ }^{\text {th }}$ Ed.). AOAC International.

Bertrand, M. E., St-Gelais, D., \& Turgeon, S. L. (2008). Characterization of acid milk gels containing protein-polysaccharide complexes: rheological properties, structure and syneresis behaviour [Tesis Doctoral, no publicada]. Universidad Laval.

Céspedes, V. (2018, Octubre 10-11). Lácteos en Centroamérica como oportunidad para crecer [Conferencia oral]. VII Congreso Centroamericano del Sector Lácteo 2018 Costa Rica, San José, Costa Rica. http://proleche.com/wp-content/ uploads/2018/10/CL2018SVA1.pdf

El-Fattah, A. A., Sakr, S., El-Dieb, S., \& Elkashef, H. (2018). Developing functional yogurt rich in bioactive peptides and gamma-aminobutyric acid related to cardiovascular health [Desarrollando un yogur functional rico en péptidos bioactivos y ácido gama-aminobutírico relacionado con la salud cardiovascular]. LWT- Food Science and Technology, 98, 390-397. https://doi.org/10.1016/j.lwt.2018.09.022

González, J. (2013, Diciembre 4-5). Situación actual y perspectivas del sector lácteo costarricense. Visión de la cámara nacional de productores de leche [Conferencia oral]. Congreso Nacional Lechero, San Carlos, Costa Rica. http:// www.proleche.com/recursos/documentos/congreso2013/Situacion_actual_y_perspectivas_del_sector_lacteo_a_ nivel_nacional_Vision_de_la_Camara_Lic_Jorge_Manuel_Gonzalez_Echeverria_Costa_Rica.pdf

González-Martínez, C., Becerra, M., Cháfer, M., Albors. A., Carot, J. M., \& Chiralt, A. (2002). Influence of substituting milk powder for whey powder on yoghurt quality. Trends in Food Science \& Technology, 13(9-10), 334-340. https://doi. org/10.1016/S0924-2244(02)00160-7

González-Olivares, L., Jiménez-Guzmán, J., Cruz-Guerrero, A., Rodríguez-Serrano, G., Gómez-Ruiz, L., \& García-Garibai, L. (2011). Liberación de péptidos bioactivos por bacterias lácticas en leches fermentadas comerciales. Revista Mexicana de Ingeniería Química, 10(2), 179-188.

Hugunin, A. (2009). U.S. Whey ingredients in yogurt and yogurt beverages. U.S. Dairy Export Council. https://www. thinkusadairy.org/Documents/Customer\%20Site/C3-Using\%20Dairy/C3.7-Resources\%20and\%20Insights/03Application\%20and\%20Technical\%20Materials/YOGURT_ENG.pdf 
Instituto Nacional de Estadística y Censos. (2014). Encuesta Nacional de Ingresos y Gastos de Hogares. http://www.inec.go.cr/ documento/enigh-2013-principales-resultados

Madriz, J. A. (2017, Octubre 10-11). Sector lácteo costarricense en el marco de la apertura comercial ¿Qué nos espera? [Conferencia oral]. XXIII Congreso Nacional Lechero, San José, Costa Rica. http://proleche.com/wp-content/ uploads/2017/10/Charla1.pdf

Mathai, J. K., Liu, Y., \& Stein, H. H. (2017). Values for digestible indispensable amino acid scores (DIAAS) for some dairy and plant proteins may better describe protein quality than values calculated using the concept for protein digestibilitycorrected amino acid scores (PDCAAS). British Journal of Nutrition, 117(4), 490-499. https://doi.org/10.1017/ S0007114517000125

Matumoto-Pintro, P. T., Rabiey, L., Robitaille, G., \& Britten, M. (2011). Use of modified whey protein in yogurt formulations. International Dairy Journal, 21(1), 21-26. https://doi.org/10.1016/j.idairyj.2010.07.003

Michelke, L., Deussen, A., Dieterich, P., \& Martin, M. (2017). Effects of bioactive peptides encrypted in whey-, soy- and rice protein on local and systemic angiotensin-converting enzyme activity. Journal of Functional Foods, 28, 299-305. https://doi.org/10.1016/j.jff.2016.11.026.

Ministerio de Economía, Industria y Comercio. (2008). Decreto $N^{\circ}$ 19091. La Gaceta. https://www.imprentanacional.go.cr/ pub/2009/03/10/COMP_10_03_2009.html

Parra, R. A. (2009). Lactosuero: importancia en la industria de alimentos. Revista Facultad Nacional de Agronomía de Medellín, 62(1), 4967-4982.

Prazeres, A. R., Carvalho, F., \& Rivas, J. (2012). Cheese whey management: A Review. Journal of Environmental Management, 110, 48-68. https://doi.org/10.1016/j.jenvman.2012.05.018

Rojas-Castro, W. N., Chacón-Villalobos, A., \& Pineda-Castro, M. L. (2007). Características del yogurt batido de fresa derivadas de diferentes proporciones de leche de vaca y cabra. Agronomía Mesoamericana, 18(2), 221-237. https://doi. org/10.15517/AM.V18I2.5052

Smithers, G. W. (2015). Whey-ing up the options - Yesterday, today and tomorrow. International Dairy Journal, $48,2-14$. https://doi.org/10.1016/j.idairyj.2015.01.011

Sodini, I., Mattas, J., \& Tong, P. S. (2006). Influence of pH and heat treatment of whey on the functional properties of whey protein concentrates in yoghurt. International Dairy Journal, 16(12), 1464-1469. https://doi.org/10.1016/j. idairyj.2005.03.014

Sodini, I., Montella, J., \& Tong, P. S. (2005). Physical properties of yogurt fortified with various commercial whey protein concentrates. Journal of the Science of Food and Agriculture, 85(5), 853-859. https://doi.org/10.1002/jsfa.2037

Sousa, G. T., Lira, F. S., Rosa, J. C., de Oliveira, E. P., Oyama, L. M., Santos, R. V., \& Pimentel, G. D. (2012). Dietary whey protein lessens several risk factors for metabolic diseases: a review. Lipids in Health Disease, 11, Article 67. https:// doi.org/10.1186/1476-511X-11-67

Sullivan, D. M, \& Carpenter, D. E. (Eds.) (1993). Methods of analysis for nutrition labeling. AOAC International.

Tetra Pak. (Ed.). (2015). Dairy Processing Handbook. Tetra Pak. https://dairyprocessinghandbook.com 
Unal, G., \& Akalın, A. S. (2012). Antioxidant and angiotensin-converting enzyme inhibitory activity of yoghurt fortified with sodium calcium caseinate or whey protein concentrate. Dairy Science \& Technology, 92, 627-639. https://doi. org/10.1007/s13594-012-0082-5

U.S. Dairy Export Council. (s.f.). Reference manual for U.S whey and lactose products. http://usdec.files.cms-plus.com/ PDFs/2008ReferenceManuals/Whey_Lactose_Reference_Manual_Complete2_Optimized.pdf

Walzem, R. L., Dillard, C. J., \& German, J. B. (2002). Whey components: millennia of evolution create functionalities for mammalian nutrition: what we know and what we may be overlooking. Critical Reviews in Food Science and Nutrition, 42(4), 353-375. https://doi.org/10.1080/10408690290825574

Zare, F., Boye, J. J., Orsat, V., Champagne, C., \& Simpson, B. K. (2011). Microbial, physical and sensory properties of yogurt supplemented with lentil flour. Food Research International, 44(8), 2482-2488. https://doi.org/10.1016/j. foodres.2011.01.002 